\title{
Buffer-Aided Relay Selection for Cooperative Diversity Systems without Delay Constraints
}

\author{
Ioannis Krikidis, Member, IEEE, Themistoklis Charalambous, Member, IEEE, \\ and John S. Thompson, Member, IEEE
}

\begin{abstract}
In this paper, we study the relay selection problem for a finite buffer-aided decode-and-forward cooperative wireless network. A relay selection policy that fully exploits the flexibility offered by the buffering ability of the relay nodes in order to maximize the achieved diversity gain is investigated. This new scheme incorporates the instantaneous strength of the wireless links as well as the status of the finite relay buffers and adapts the relay selection decision on the strongest available link by dynamically switching between relay reception and transmission. In order to analyse the new relay selection policy in terms of outage probability and diversity gain, a theoretical framework that models the evolution of the relay buffers as a Markov chain (MC) is introduced. The construction of the state transition matrix and the related steady state of the MC are studied and their impact on the derivation of the outage probability is investigated. We show that the proposed relay selection scheme significantly outperforms conventional relay selection policies for all cases and ensures a diversity gain equal to two times the number of relays for large buffer sizes.
\end{abstract}

Index Terms-Cooperative networks, relay channel, buffers, relay selection, outage probability, Markov chain.

\section{INTRODUCTION}

$\mathbf{R}$ ECENTLY cooperative transmission has received a lot of attention because it can enhance the reliability of data transmission, provide broader and low-cost coverage and combat shadowing and fading effects [1]. For cooperative networks with multiple relay nodes that can assist the source transmission through either multi-hop relaying or cooperative diversity, relay selection has been introduced as an efficient cooperative technique. More precisely, relay selection allows a single relay node (the best node according to some well defined criterion) to participate in the cooperation process and has been proven to use more efficiently the system resources (e.g., power, bandwidth) without affecting the system performance. The main advantage of relay selection is the implementation simplicity, as it does not require complex physical layer transmission techniques (e.g., distributed spacetime codes) or explicit synchronization processes. Since the seminal work of Bletsas et al. [2] where the fundamental

Manuscript received November 5, 2011; accepted February 15, 2012. The associate editor coordinating the review of this paper and approving it for publication was G. Song.

I. Krikidis is with the Department of Computer Engineering and Informatics, University of Patras, Greece (e-mail: krikidis@ucy.ac.cy).

T. Charalambous is with the Department of Electrical Engineering, KTH Royal Institute of Technology, Stockholm, Sweden (e-mail: themisc@kth.se).

J. S. Thompson is with the Institute for Digital Communications (IDCOM), University of Edinburgh, Edinburgh EH9 3JL, UK (e-mail: john.thompson@ed.ac.uk).

Digital Object Identifier 10.1109/TWC.2012.032712.111970 benefits from the relay selection have been introduced, a flood of papers has appeared on this subject and many relay selection policies have been proposed for different cooperative contexts (see for example [3]-[7] and references therein).

Although relay selection is introduced as a general radio design technique for several cooperative applications with different optimisation targets, in this paper we re-examine its basic form. More specifically, we focus on a simple decodeand-forward (DF) cooperative network where a source communicates with a destination through a set of half-duplex relay nodes with the objective of performance optimisation. For this fundamental problem, the max - min relay selection scheme [2], [8], [9], where the selected relay holds the strongest end-to-end relaying path, achieves the optimal performance and ensures a full diversity equal to the number of the relays. However, this relay selection policy refers to a two-slot cooperative transmission where the selected relay receives the source's data during the first time slot and immediately in the next time slot forwards the data towards the destination. In order to overcome this limitation imposed by the max - min selection scheme, recently in [10] the authors introduced data buffers at the relay nodes that allow the selection of a different relay for reception and transmission in order to extract further diversity gains. In that work, the max - max relay selection scheme that selects the relay with the strongest source-relay channel and the strongest relay-destination channel for reception and transmission, respectively, has been investigated. The max - max scheme refers to applications without critical delay constraints and provides a significant coding gain in comparison to the conventional max - min selection scheme. However, despite the efficient use of the channel fading and the related performance benefits, the max - max relay selection policy requires a predefined schedule for the relaying transmission (e.g., the second slot is always allocated for relaying) and therefore the available diversity degrees are not fully exploited. It is worth noting that the use of buffers in order to boost the achieved performance (in terms of throughput) has been reported in the literature in different contexts [11]-[16]. In [16], the authors investigate a relay selection policy for a cooperative system with finite buffers at the relays and analyze the system in terms of throughput; the selection criterion is based on the maximization of the achievable rate and extended numerical results demonstrate the impact of the buffer size on the system performance.

In this paper, we consider a cooperative network with buffers of finite size at the relay nodes and we try to overcome 
the above limitation of the max - max relay selection policy. The main outcome of this work is a new relay selection scheme that fully exploits the available diversity degrees offered by the wireless channels through intelligent and dynamic switching between source and relay transmission. The corresponding selection scheme is referred to as max - link relay selection and at each slot the relay with the strongest available link is selected either for transmission or reception. The relay selection decision incorporates the instantaneous quality of the wireless links as well as the status of the finite relay buffers (e.g., full, empty, neither full nor empty) and adapts the slot allocation accordingly. In order to analyse the proposed selection scheme in terms of outage probability, a theoretical framework that models the evolution of the relay buffers as a state Markov chain (MC) is investigated. By using as theoretical tools the state transition matrix and the related steady state of the MC, the outage probability of the system is derived in closed form. We prove that the max - link relay selection achieves a significant coding gain for the low buffer sizes while it provides diversity gains as the buffer size increases. In addition, for high (but finite) buffer sizes, it achieves a full diversity gain that it is equal to two times the number of the relays; this result makes the proposed scheme an attractive and practical solution for applications without latency constraints. It is worth noting that the max - link relay selection scheme handles the cases that finite-relay buffers are full or empty without any structural modification; previous solutions overcome these cases either by changing the protocol in use [10, Sec. II. D] or by assuming infinite buffers at the relays [12]. In addition, the main difference of our study with the related work in [16], is that here, we study a buffer-aided relay scheme from a diversity standpoint (extraction of the maximum diversity gain), whereas in [16] the selection policy maximizes the achievable throughput. Finally, a theoretical framework for the analysis of the outage performance is presented in this paper for the first time, while previous studies ([10], [16]) are mainly based on simulation results.

The contribution of the paper is three-fold:

- We extend the work presented in [10] by integrating the relay selection problem and we study the system from a diversity perspective using outage probabilities.

- We present a theoretical framework for the analysis of the system based on a Markov chain formulation.

- We derive closed-form expressions for the achieved outage performance of the system for finite and infinite buffer sizes.

Notation: In this paper, we use boldface lower case letters to denote vectors and boldface capital letters to denote matrices. Further, $(\cdot)^{-1}$ and $(\cdot)^{T}$ stand for matrix inversion and transposition, respectively, $\operatorname{diag}(\cdot)$ returns the diagonal of a matrix, I denotes the identity matrix (with appropriate dimensions) and $\mathbf{O}_{m \times n}$ is a $m \times n$ matrix with all elements equal to zero. $A_{m, \bullet}$ and $A_{\bullet, m}$ denote the $m$-th row and column of a matrix $\mathbf{A}$, respectively. $\mathbf{A}_{i, j}$ is the $(i, j)$ entry of $\mathbf{A}$. The sets of real and natural numbers are denoted by $\mathbb{R}$ and $\mathbb{N}$, respectively; their positive orthant is denoted by the subscript + (e.g., $\mathbb{R}_{+}$). Finally, $\mathbb{P}(X)$ denotes the probability of event $X$.

The remainder of the paper is organised as follows. Section II introduces the system model and presents the basic

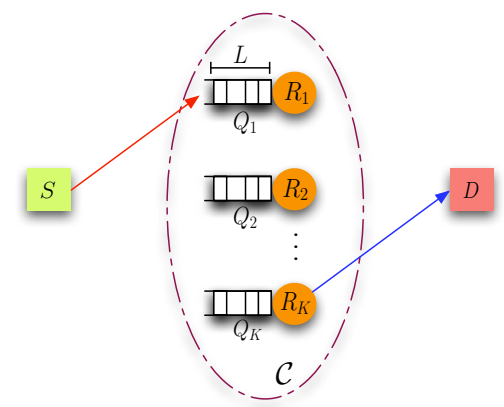

Fig. 1. The system model: source $S$ communicates with Destination $D$ via a cluster of relays $R_{k} \in \mathcal{C}(1 \leq k \leq K)$.

assumptions made in this study. Section III reviews the main previously reported relay selection schemes and introduces the max - link relay selection policy. The state Markovian channel model and the theoretical framework for the computation of the outage probability is presented in Section IV. Some illustrative examples are also presented in Section $\mathrm{V}$ for better visualisation of the proposed scheme, whereas numerical results are shown and discussed in Section VI, followed by concluding remarks in Section VII.

\section{SySTEM MODEL}

We assume a simple cooperative network consisting of one source $S$, one destination $D$ and a cluster $\mathcal{C}$ with $K$ DF relays $R_{k} \in \mathcal{C}(1 \leq k \leq K)$. All nodes are characterised by the half-duplex constraint and therefore they cannot transmit and receive simultaneously. A direct link between the source and the destination does not exist (the channel gain between source and destination is in deep fading) and communication can be established only via relays (i.e., [2], [5], [10]). Each relay $R_{k}$ holds a data buffer (data queue) $Q_{k}$ of finite size $L$ (in number of data elements) where it can store data coming from the source that have been decoded at the relay and can be forwarded to the destination. Fig. 1 schematically presents the system model.

At the beginning of the transmission each relay buffer is empty and the function $0 \leq \Psi\left(Q_{k}\right) \leq L$ gives the number of data elements that are stored in buffer $Q_{k}$. The buffer size $\Psi\left(Q_{k}\right)$ is increased by one when a source packet (data) is correctly decoded at the $k$-th relay and it is decreased by one in case of a successful $k$-th relay transmission. Time is considered to be slotted and each transmitter $X$ (either the source $S$ or relays $R_{k}$ ) transmits with a fixed power $P$. The source node is assumed to be saturated (infinite data backlog at the source) and the information rate is equal to $r_{0}$ bits per channel use (BPCU). The retransmission process is based on an Acknowledgement/Negative-Acknowledgement (ACK/NACK) mechanism, in which short-length error-free packets are broadcasted by the receivers $Y$ (either a relay $R_{k}$ or the destination $D$ ) over a separate narrow-band channel in order to inform the network of that packet's reception status.

In order to simplify the analysis ${ }^{1}$, we assume that the clustered relay configuration ensures independent and iden-

\footnotetext{
${ }^{1}$ The proposed analysis does not depend on the i.i.d. assumption and is used for better exposition only. The i.i.d. case and the related analysis can be considered as a useful guideline for more complex asymmetric configurations.
} 
tically distributed (i.i.d.) channel links that means equivalent average Signal-to-Noise Ratios (SNRs) for the links $S \rightarrow R_{k}$ and $R_{k} \rightarrow D$ [17]. Although this assumption simplifies the analysis and it is used in several studies in the literature [18]-[21], it can be implemented by an appropriate longterm routing process which selects the clustered nodes to be closed together [19], [20]. All wireless links exhibit fading and Additive White Gaussian Noise (AWGN). The fading is assumed to be stationary, with frequency non-selective Rayleigh block fading. This means that the fading coefficients $h_{i, j}$ (for the $i \rightarrow j$ link) remain constant during one slot, but change independently from one slot to another according to a circularly symmetric complex Gaussian distribution with zero mean and unit variance. Furthermore, the variance of the AWGN is assumed to be normalised with zero mean and unit variance and therefore the SNR for each link is equal to $P$. Each link $i \rightarrow j$ is characterised by the success probability $p \triangleq \mathbb{P}\left\{(1 / 2) \log _{2}\left(1+P\left|h_{i, j}\right|^{2}\right) \geq r_{0}\right\}$ which denotes the probability that the link $i \rightarrow j$ is not in outage $(\bar{p}=1-p$ denotes the outage probability); the factor $1 / 2$ captures the fact that communication is performed in two time slots [1], [10]. An outage occurs when the instantaneous capacity of the link $i \rightarrow j$ is lower than the transmitted spectral efficiency rate $r_{0}$. Finally, we assume that the destination node has a perfect channel and buffer state information and selects the relays for transmission and reception through an error-free feedback channel[10]. This assumption can be ensured by an appropriate signalling that provides global channel state information (CSI) at the destination node. On the other hand, the destination can trivially know the status of the relay buffers by monitoring the ACK/NACK signalling and the identity of the transmitting/receiving relay. It is worth noting that our focus is to investigate the performance of a new buffer-aided relay selection scheme under the global CSI assumption and hence, the implementation issues (i.e., distributed implementations [2], CSI acquisition, etc.) are beyond the scope of this paper.

\section{Relay Selection Policies}

In this Section, we briefly review the main selection policies used in the literature and thereafter we introduce our suggested relay selection policy, called the max - link selection policy.

\section{A. $\max -\min$ relay selection}

The max - min relay selection policy [2], [9] is considered as the optimal selection scheme for conventional DF relay setups without buffers and is used as our baseline relay selection strategy. The selected relay is the one that provides the "best" (strongest) end-to-end path between the source and the destination. The max - min selection policy ensures a full diversity (equal to the number of the relays (e.g., $K)$ ) and refers to a standard cooperative protocol where communication is performed in two time slots: a) in the first time slot, the source transmits b) in the second time slot, the selected relay forwards the data received by the source towards the destination. The $\max -\min$ relay selection policy can be written as

$$
R^{*}=\arg \max _{R_{k} \in \mathcal{C}} \min \left\{\left|h_{S, R_{k}}\right|^{2},\left|h_{R_{k}, D}\right|^{2}\right\} .
$$

where $R^{*}$ denotes the selected relay. Although the max - min selection scheme achieves a diversity equal to $K$, it does not take into account the fact that relays are equipped with buffers and their potential benefits. The selected relay forwards the received data (in case of a successful decoding) immediately in the next time slot to the destination and therefore the ability of the relays to store at least a limited number of data packets is not elaborated.

\section{B. $\max -\max$ relay selection (bound)}

The max - max relay selection [10] is the first policy that considers the buffering capability at the relay nodes and is used as a reference selection scheme. Given that the relay nodes are equipped with buffers and thus can store the data received from the source, the max - max policy selects the end-to-end path that holds the strongest source-relay and relay-destination links. More specifically, it splits the relay selection decision in two parts and selects the relay with the best source-relay link for reception and the relay with the best relay-destination link for transmission. The max - max selection policy respects the conventional two-slot cooperative transmission where the first slot is dedicated for the source transmission and the second slot for the relaying transmission, but the relay node may not be the same for both phases of the protocol. The max - max relay selection policy can be written as

$$
R_{r}^{*}=\arg \max _{R_{k} \in \mathcal{C}}\left\{\left|h_{S, R_{k}}\right|^{2}\right\}, \quad R_{t}^{*}=\arg \max _{R_{k} \in \mathcal{C}}\left\{\left|h_{R_{k}, D}\right|^{2}\right\},
$$

where $R_{r}^{*}$ and $R_{t}^{*}$ denote the relay selected for the first phase and the second phase of the cooperative protocol, respectively. It has been proven that the max - max relay selection policy also ensures full diversity equal to the number of the relays and it provides a significant coding gain in comparison to the conventional max - min selection scheme. However, it is worth noting the the above selection strategy assumes that no relay's buffer can be empty or full at any time and thus all relays have always the option of receiving or transmitting [10, Sec. III. C]. Therefore, the $\max -\max$ relay selection considered provides the optimal performance that can be achieved by the max - max relay selection scheme and yields the lowest outage bound.

\section{C. $\max -$ link relay selection}

The previous relay selection schemes are associated with a two-slot cooperative protocol where the schedule for the source and relay transmission is fixed a priori. Here, we relax this limitation and we allow each slot to be allocated dynamically to the source or a relay transmission, according to the instantaneous quality of the links and the status of the relays' buffers. More specifically, the proposed max - link relay selection scheme exploits fully the flexibility offered by the buffers at the relay nodes and at each slots it selects the strongest link for transmission (source or relay transmission) among the available links. A source-relay link is considered to be available when the corresponding relay node is not full and therefore can receive data from the source, while a relay-destination link is considered to be available when the 
relay node is not empty and thus can transmit source's data towards the destination. The proposed scheme compares the quality of the available links and adjusts the relay selection decision and the time slot allocation to the strongest link. If a source-relay link is the strongest link, the source transmits and the corresponding relay is selected for reception; on the other hand, if a relay-destination link is the strongest link, the corresponding relay is selected for transmission. The max - link relay selection policy can be analytically expressed as follows:

$$
\begin{array}{r}
R^{*}=\arg \max _{R_{k} \in \mathcal{C}}\left\{\bigcup_{R_{k} \in \mathcal{C}: \Psi\left(Q_{k}\right) \neq L}\left\{\left|h_{S, R_{k}}\right|^{2}\right\}\right. \\
\left.\bigcup_{R_{k} \in \mathcal{C}: \Psi\left(Q_{k}\right) \neq 0}\left\{\left|h_{R_{k}, D}\right|^{2}\right\}\right\},
\end{array}
$$

where $R^{*}$ denotes the selected relay (either for transmission or reception).

For the max - link relay selection policy the outage probability is defined as the probability that the selected link is in outage, i.e.,

$$
P_{\text {out }} \triangleq\left\{\begin{array}{l}
\mathbb{P}\left(\frac{1}{2} \log _{2}\left(1+P\left|h_{S, R^{*}}\right|^{2}\right)<r_{0}\right) \\
\text { for relay reception; } \\
\mathbb{P}\left(\frac{1}{2} \log _{2}\left(1+P\left|h_{R^{*}, D}\right|^{2}\right)<r_{0}\right) \\
\text { for relay transmission. }
\end{array}\right.
$$

In order to be able to analyse the max - link relay selection policy, we model the possible states of the buffers and the transitions between the states as a MC. A state of the MC represents the number of elements at each buffer and thus $s_{l} \triangleq\left(\Psi\left(Q_{1}\right) \Psi\left(Q_{2}\right) \ldots \Psi\left(Q_{K}\right)\right)$ denotes the $l$-th state of the MC with $l \in \mathbb{N}_{+}, 1 \leq l \leq(L+1)^{K}$. The states are predefined in a random way as all the possible combinations $\left((L+1)^{K}\right.$ combinations) of the buffer sizes and are considered as a data input for the investigated algorithm given in Section IV. The use of the MC model for the analysis of the max - link selection policy in terms of outage probability is presented in the next section.

\section{The max - link Relay Selection: Model and OUtage Probability ANALYSIS}

In this Section, we deal with the outage probability analysis of the max - link relay selection policy and we introduce a theoretical framework that constructs the state transition matrix and calculates the outage probabilities (this performance metric is also used for the evaluation of the $\max -\max$ relay selection in [10]). The selected performance metric is appropriate for environments with multipath and fading effects where diversity order becomes a critical performance parameter. In addition, we note that the main target of relay selection in this paper is to extract diversity gain and therefore the outage probability metric is mainly used in the related literature i.e., [4], [5], [8], [9]. The investigation of a theoretical tool for the analysis of the max - link selection with finite buffers is a main result of this paper and is introduced as a general methodology to analyze systems with finite buffers.

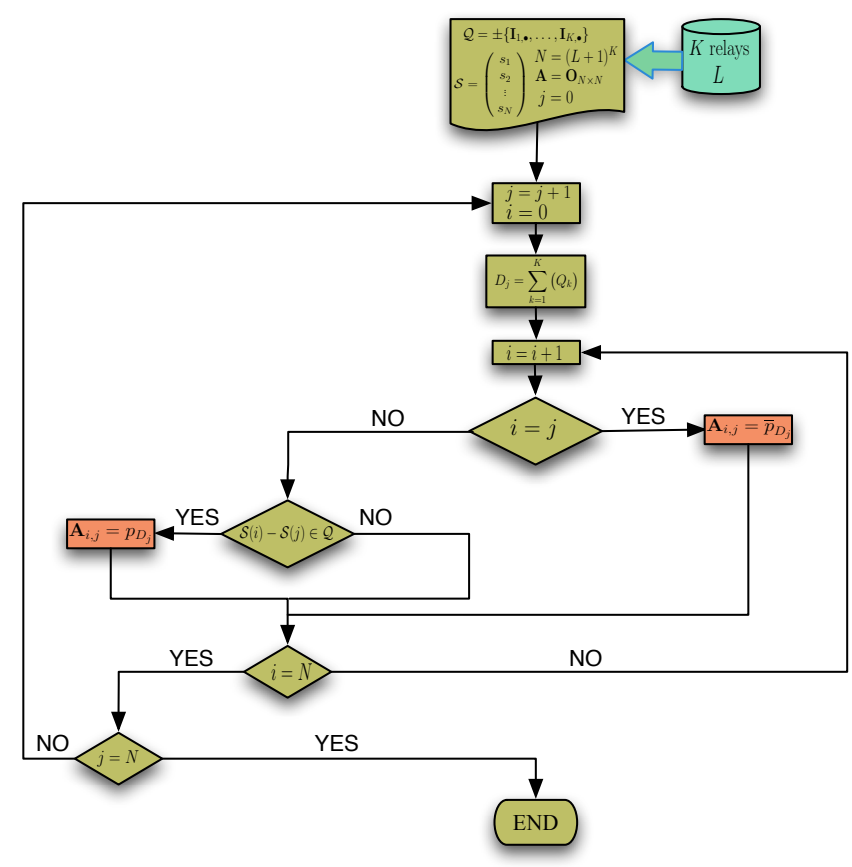

Fig. 2. Flow diagram for the generation of the state transition matrix A.

\section{A. Construction of the state transition matrix of the $M C$}

The state transition matrix represents the MC of the buffers' states and models the connectivity between them. It is a key element of the proposed analytical framework and its construction is fundamental for the computation of the outage probabilities. Fig. 2 depicts the block diagram for the construction of the state transition matrix based on the system parameters $K, L$. Let A denote the $(L+1)^{K} \times(L+1)^{K}$ state transition matrix of the $\mathrm{MC}$, in which the entry $\mathbf{A}_{i, j}=\mathbb{P}\left(s_{j} \rightarrow s_{i}\right)=$ $\mathbb{P}\left(X_{t+1}=s_{i} \mid X_{t}=s_{j}\right)$ is the transition probability to move from state $s_{j}$ at time $t$ to state $s_{i}$ at time $(t+1)$. The transition probabilities depend on the status of the relay buffers (number of full elements at each buffer) and the related number of the available links that participate in the relay selection decision. More specifically, a relay node with a full or empty data buffer $\left(\Psi\left(Q_{k}\right)=L\right.$ or $\left.\Psi\left(Q_{k}\right)=0\right)$ cannot receive or transmit data, respectively, and therefore for both cases it offers only one link in the selection process; when $\Psi\left(Q_{k}\right)=L$ the $k$-th relay offers the link $R_{k} \rightarrow D$ and when $\Psi\left(Q_{k}\right)=0$ it offers the link $S \rightarrow R_{k}$. Otherwise, the relay node can be used either for transmission or reception and thus it offers two links for selection $\left(S \rightarrow R_{k}, R_{k} \rightarrow D\right)$. Consequently, for the $s_{l}$ state of the buffers, the total number of the available links that participate in the max - link selection process is equal to

$$
D_{l}=\sum_{i=1}^{K} \Phi\left(Q_{i}\right)
$$

where

$$
\Phi\left(Q_{i}\right)= \begin{cases}2 & \text { if } 0<\Psi\left(Q_{i}\right)<L \\ 1 & \text { elsewhere }\end{cases}
$$

In order to construct the state transition matrix $\mathbf{A}$, we need to identify the connectivity between the different states of the buffers. For each time slot, the buffer status can be modified as 
follows: (a) the number of elements of one relay buffer can be decreased by one, if a relay node is selected for transmission and the transmission is successful, (b) the number of elements of one buffer can be increased by one, if the source node is selected for transmission and the transmission is successful and (c) the buffer status remains unchanged in case of outage (e.g., either the source has been selected for transmission and the selected source-relay link was in outage or a relay has been selected for transmission and the relay-destination link was in outage). In order to formulate the above buffer state connectivity, we define for the $s_{l}$ state the associated set $U_{l}$ given by

$$
U_{l}=\left\{\bigcup_{1 \leq i \leq(L+1)^{K}} s_{i}: \mathbf{s}_{i}-\mathbf{s}_{l} \in \mathcal{Q}\right\},
$$

where $\mathbf{s}_{l}$ denotes a $1 \times K$ vector with entries the number of elements stored at each buffer for the $s_{l}$ state and $\mathcal{Q} \triangleq$ $\left\{\bigcup_{1 \leq j \leq K} \pm \mathbf{I}_{j, \bullet}\right\}$. The set $U_{l}$ contains all the buffer states that are connected to the state $s_{l}$ based on the previous connectivity rule. As for the computation of the corresponding transition probabilities, we should take into account that a transition from one state to another one requires a) the selection of the corresponding channel link by the max - link selection process and b) the selected link not be in outage (e.g., successful transmission). Given our assumption for i.i.d. symmetric channel links, for the $s_{l}$ buffer state, the probability to select a specific link is equal to $1 / D_{l}$ and the probability that the selected link is not in outage can be calculated by using order statistics (the maximum among $D_{l}$ i.i.d. exponential random variables); therefore the probability to leave from the state $s_{l}$ is equal to

$$
p_{D_{l}} \triangleq \frac{1}{D_{l}}\left[1-\left(1-\exp \left(-\frac{2^{2 r_{0}}-1}{P}\right)\right)^{D_{l}}\right]
$$

On the other hand the probability to have an outage event and therefore no change in the buffer status is equal to

$$
\bar{p}_{D_{l}} \triangleq 1-\sum_{i=1}^{D_{l}} p_{D_{l}}=\left(1-\exp \left(-\frac{2^{2 r_{0}}-1}{P}\right)\right)^{D_{l}} .
$$

By using the previous notation, the entries of the state transition matrix are given as

$$
\mathbf{A}_{i, j}=\left\{\begin{array}{l}
\bar{p}_{D_{j}} \text { if } s_{i} \notin U_{j} \\
p_{D_{j}} \text { if } s_{i} \in U_{j} \quad, \quad \text { for } i, j \in\left\{1, \ldots,(L+1)^{K}\right\} \\
0 \text { elsewhere }
\end{array}\right.
$$

\section{B. Steady state distribution of the $M C$}

We are interested in finding the stationary distribution, denoted as $\pi$, of the MC and hence, explore how the data are being sent across the relays and the long-term share of the resources.

Proposition 1. The state transition matrix $\mathbf{A}$ of the $M C$ that models the buffer states is a column stochastic matrix ${ }^{2}$.

\footnotetext{
${ }^{2} \mathrm{~A}$ column stochastic matrix is a square matrix for which the sum of the elements in each of its columns is 1 [22].
}

Proof: For any MC the transition from state $s_{j}$ to $s_{i}$ for all possible states $s_{j}$ sums up to 1, i.e.,

$$
\sum_{i=1}^{(L+1)^{K}} \mathbf{A}_{i, j}=1
$$

If there exists a transition from state $s_{i}$ to $s_{j}$, then there exists a transition from state $s_{j}$ to $s_{i}$. However, since the states are not symmetric and the number of links to other states is not the same, the transition probabilities are not the same. Thus, the transition matrix $\mathbf{A}$ is not symmetric. As a result, the transition probability is column stochastic only and not doubly stochastic.

Proposition 2. The state transition matrix $\mathbf{A}$ of the $M C$ that models the buffer states is irreducible ${ }^{3}$ and aperiodic ${ }^{4}$.

Proof: Due to the structure of the problem all the possible states of the MC considered can communicate and hence its state space is a single communicating class; in other words, it is possible to get to any state from any state. Hence, the MC is irreducible. Also, the probability of being at any state after $N$ and $N+1$ transitions is greater than zero; hence, all states are aperiodic and therefore, the MC is aperiodic.

Proposition 3. The state transition matrix $\mathbf{A}$ of the $M C$ that models the buffer states is reversible $e^{5}$.

Proof: Since the MC is irreducible and the edges from a state $i$ to any other state $j$ are equal, and given that every vertex has a finite valency ${ }^{6}$, then $\boldsymbol{\pi}$ is invariant and $\mathbf{A}$ is reversible.

Lemma 1. The stationary distribution of the column stochastic matrix $\mathbf{A}$ of the MC that models the buffer states is given by

$$
\boldsymbol{\pi}=(\mathbf{A}-\mathbf{I}+\mathbf{B})^{-1} \mathbf{b}
$$

where $\boldsymbol{\pi}$ is the stationary distribution, $\boldsymbol{b}=\left(\begin{array}{llll}1 & 1 & \ldots & 1\end{array}\right)^{T}$ and $\mathbf{B}_{i, j}=1, \forall i, j$.

\section{Proof: See Appendix A.}

Due to the fact that the number of states of the Markov Chain increases very rapidly, it is desirable to find an efficient method to find the stationary distribution with reduced complexity. Also, we observed that the states with equal outage probability have the same steady state probability, due to the symmetry of the problem. In Lemma 2, the symmetry arising due to the structure of the problem as well as the reversibility property (Proposition 3) are exploited in order to find an efficient way to calculate the steady states of the Markov Chain.

\footnotetext{
${ }^{3} \mathrm{~A} \mathrm{MC}$ is irreducible if and only if all states communicate [22].

${ }^{4} \mathrm{~A} \mathrm{MC}$ is aperiodic if all states are aperiodic; a state is aperiodic if and only if there exists a time $N$ such that $\mathbf{A}_{i, i}^{(N)}>0$ and $\mathbf{A}_{i, i}^{(N+1)}>0$ [22].

${ }^{5} \mathrm{~A} \mathrm{MC}$ is said to be reversible if there is a probability distribution over states, $\boldsymbol{\pi}$, such that $\boldsymbol{\pi}_{i} \mathbb{P}\left(X_{t+1}=j \mid X_{t}=i\right)=\boldsymbol{\pi}_{j} \mathbb{P}\left(X_{t+1}=i \mid X_{t}=j\right)$ for all times $t$ and all states $i$ and $j$. In other words, a MC is said to be reversible if $\left(X\left(\left(t_{1}\right) X\left(\left(t_{2}\right) \ldots X\left(\left(t_{k}\right)\right)\right.\right.\right.$ has the same distribution as $\left(X\left(\left(\tau-t_{1}\right) X((\tau-\right.\right.$ $\left.t_{2}\right) \ldots X\left(\left(\tau-t_{k}\right)\right)$ for all $\tau \geq 1$ [22].

${ }^{6}$ The valency of a vertex $i$ is the number of edges at $i$ [22].
} 


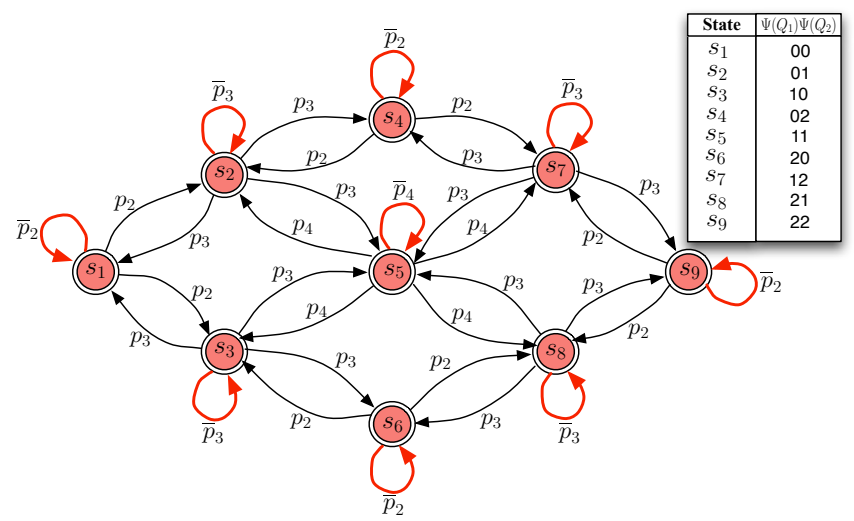

Fig. 3. State diagram of the MC representing the states of the buffers and the transitions between them for a case with $K=2$ relays and $L=2$.

Let $\Theta_{i}$ denote the set of states $i$ for which the steady state probability is the same. Let $\Xi\left(j, \Theta_{i}\right)$ denote the states that state $j$ has to pass through to reach a state $i \in \Theta_{i}$, with the state in $\Theta_{i}$ included; i.e., if from state $i$ to a state that belongs in $\Theta_{j}$, say $l$, we go through a state $k$, then $\Xi\left(i, \Theta_{j}\right)=\{k, l\}$.

Lemma 2. The steady state for each state $i, i \in \Theta_{i}$, is given by

$$
\boldsymbol{\pi}_{i}=\left(\sum_{j=1}^{(L+1)^{K}} \frac{\prod_{k \in \Xi\left(i, \Theta_{j}\right)} \mathbf{A}_{i, k}}{\prod_{m \in \Xi\left(j, \Theta_{i}\right)} \mathbf{A}_{j, m}}\right)^{-1}
$$

Proof: See Appendix B.

\section{Derivation of the outage probability}

By using the steady state of the MC and the fact that an outage event occurs when there is no change in the buffer status, the outage probability of the system can be expressed as

$$
P_{\text {out }}=\sum_{i=1}^{(L+1)^{K}} \boldsymbol{\pi}_{i} \bar{p}_{D_{i}}=\operatorname{diag}(\mathbf{A}) \boldsymbol{\pi}
$$

The above expression shows that the construction of the state matrix $\mathbf{A}$ and the computation of the related steady state $\pi$ consists of a simple theoretical framework for the computation of the outage probability for the max - link relay selection scheme with finite buffers. For the case of large $L$, a simplified expression of the above outage probability is proposed in Section V-B.

\section{Illustrative ExAmples}

In order to visualise the proposed approach for the computation of the achieved outage probability, we apply the proposed analysis to special, illustrative cases. Firstly, we include the simple case in which we have two relays $(K=2)$ with maximum buffer size equal to two $(L=2)$. Then, we consider the case for which the buffer size is infinitely large $(L \rightarrow \infty)$.
A. A simple example with $K=2$ relays and $L=2$ (case of study)

In this section, we apply the proposed analysis for a simple clustered topology with $K=2$ relays and $L=2$. In this case the state MC that captures the evolution of the buffers is presented in Fig. 3 and the corresponding state transition matrix $\mathbf{A}$ becomes equal to

$$
\mathbf{A}=\left(\begin{array}{ccccccccc}
\overline{p_{2}} & p_{3} & p_{3} & 0 & 0 & 0 & 0 & 0 & 0 \\
p_{2} & \bar{p}_{3} & 0 & p_{2} & p_{4} & 0 & 0 & 0 & 0 \\
p_{2} & 0 & \bar{p}_{3} & 0 & p_{4} & p_{2} & 0 & 0 & 0 \\
0 & p_{3} & 0 & \bar{p}_{2} & 0 & 0 & p_{3} & 0 & 0 \\
0 & p_{3} & p_{3} & 0 & \bar{p}_{4} & 0 & p_{3} & p_{3} & 0 \\
0 & 0 & p_{3} & 0 & 0 & \bar{p}_{2} & 0 & p_{3} & 0 \\
0 & 0 & 0 & p_{2} & p_{4} & 0 & \bar{p}_{3} & 0 & p_{2} \\
0 & 0 & 0 & 0 & p_{4} & p_{2} & 0 & \bar{p}_{3} & p_{2} \\
0 & 0 & 0 & 0 & 0 & 0 & p_{3} & p_{3} & \bar{p}_{2}
\end{array}\right)
$$

By using the method described in IV-B, the steady state of the system for different values of SNR is shown in Table I.

\section{B. Case with buffers of infinite size $(L \rightarrow \infty)$}

As presented in Section IV-A, the total number of buffer states is equal to $(L+1)^{K}$. However, from these $(L+1)^{K}$ states, $(L-1)^{K}$ states are neither full nor empty. In what follows, we show that for large $L$ the $(L-1)^{K}$ states dominate the system and for $L \rightarrow \infty$ the probability of being in one of these states approaches 1 . Hence, as the buffer size $L$ increases the MC approaches a symmetric matrix and therefore the steady state converges to a uniform distribution; as a result the steady state probabilities become equal.

We divide the states of the MC into two distinct categories: $F_{1}$ features the states in which at least one buffer is either empty or full, and, $F_{2}$ features the states in which the buffers are neither empty nor full. Since the state transition matrix $\mathbf{A}$ is reversible (Proposition 3), then it can easily be shown that the MC with states $F_{1}$ and $F_{2}$ is also reversible. Hence,

$$
\hat{\pi}_{F_{1}} \mathbb{P}\left(X_{t+1}=F_{2} \mid X_{t}=F_{1}\right)=\hat{\pi}_{F_{2}} \mathbb{P}\left(X_{t+1}=F_{1} \mid X_{t}=F_{2}\right),
$$

where $\hat{\pi}_{i}$ denotes the stationary probability for being at state $i$. Now, for large $L$ due to the symmetry of the states $i$ in $F_{2}$, we assume that their steady state probability is the same. Also, we let the steady state probability of the states $j$ in $F_{1}$ be upper bounded by the largest steady state probability in the states belonging to $F_{1}$. Let $P_{i \rightarrow j}$ denoting the transition probability from state $i$ to $j$. Then (16) can be written as

$$
\begin{aligned}
P_{F_{2} \rightarrow F_{1}} & =\frac{\hat{\boldsymbol{\pi}}_{F_{2}}}{\hat{\boldsymbol{\pi}}_{F_{1}}} P_{F_{1} \rightarrow F_{2}} \\
& \leq \frac{\left[(L+1)^{K}-(L-1)^{K}\right] \max _{j \in F_{1}} \boldsymbol{\pi}_{j}}{(L-1)^{K} \boldsymbol{\pi}_{i}} P_{F_{1} \rightarrow F_{2}} \\
& =\left(\frac{\left[(L+1)^{K}-(L-1)^{K}\right]}{(L-1)^{K}}\right) \frac{\max _{j \in F_{1}} \boldsymbol{\pi}_{j}}{\boldsymbol{\pi}_{i}} P_{F_{1} \rightarrow F_{2}} .
\end{aligned}
$$


TABLE I

StATE PRobAbILITIES FOR DifFERENT SNRS $(P) ; K=2$ RELAYs, $L=2, r_{0}=1 \mathrm{BPCU}$

\begin{tabular}{|c||c|c|c|c|}
\hline Buffer state & $\boldsymbol{\pi}(P=0 \mathrm{~dB})$ & $\boldsymbol{\pi}(P=8 \mathrm{~dB})$ & $\boldsymbol{\pi}(P=20 \mathrm{~dB})$ & $\boldsymbol{\pi}(P=30 \mathrm{~dB})$ \\
\hline$s_{1}$ & 0.1093 & 0.0894 & 0.0834 & 0.0783 \\
$s_{2}$ & 0.1120 & 0.1215 & 0.1250 & 0.1326 \\
$s_{3}$ & 0.1120 & 0.1215 & 0.1250 & 0.1326 \\
$s_{4}$ & 0.1093 & 0.0894 & 0.0834 & 0.0783 \\
$s_{5}$ & 0.1148 & 0.1564 & 0.1666 & 0.1565 \\
$s_{6}$ & 0.1093 & 0.0894 & 0.0834 & 0.0783 \\
$s_{7}$ & 0.1120 & 0.1215 & 0.1250 & 0.1326 \\
$s_{8}$ & 0.1120 & 0.1215 & 0.1250 & 0.1326 \\
$s_{9}$ & 0.1093 & 0.0894 & 0.0834 & 0.0783 \\
\hline
\end{tabular}

For the extreme case with infinite buffer sizes $L \rightarrow \infty$, the transition probability from $F_{2}$ to $F_{1}$ becomes

$$
\begin{aligned}
\lim _{L \rightarrow \infty} P_{F_{2} \rightarrow F_{1}} \leq \lim _{L \rightarrow \infty}(\underbrace{\frac{\left[(L+1)^{K}-(L-1)^{K}\right]}{(L-1)^{K}}}) \\
\quad \times \frac{\max _{j \in F_{1} \pi_{j}} \rightarrow 0}{\pi_{i}} P_{F_{1} \rightarrow F_{2}} \\
=0 .
\end{aligned}
$$

As previously mentioned, this suggests that for large $L$ the states in $F_{2}$ dominate the system. Hence, as the buffer size $L$ increases the steady state converges to a uniform distribution; as a result the steady state probabilities become equal.

Remark 1. Note that the dominance of the states in $F_{2}$ can also be seen if we consider the proportion of states for large $L$ and the fact that the probability of being in a set of states is upper bounded by a constant multiplied to the number of states in the set. Hence, by denoting by $\left|F_{i}\right|$ to be the cardinality of set $F_{i}$, the limits of the proportion of the two sets $F_{1}$ and $F_{2}$ are as follows:

$$
\begin{aligned}
& \lim _{L \rightarrow \infty} \frac{\left|F_{1}\right|}{(L+1)^{K}}=\lim _{L \rightarrow \infty} \frac{(L+1)^{K}-(L-1)^{K}}{(L+1)^{K}}=0, \\
& \lim _{L \rightarrow \infty} \frac{\left|F_{2}\right|}{(L+1)^{K}}=\lim _{L \rightarrow \infty} \frac{(L-1)^{K}}{(L+1)^{K}}=1 .
\end{aligned}
$$

This simplification yields a simple characterisation of the outage probability for large $L$ as well as for the extreme case with $L \rightarrow \infty$. Given the rule for the evolution of the relay's buffers described in Section IV-A and the approximation that the stationary distribution is uniform, then the probability for each state is equal to $1 /(L+1)^{K}$. Among these states, the total number of states that have $i(0 \leq i \leq K)$, full or empty buffers is equal to

$$
\nu_{i} \triangleq \underbrace{2^{i}\left(\begin{array}{c}
K \\
i
\end{array}\right)}_{\text {full/empty buffers }} \cdot \underbrace{(L-1)^{K-i}}_{\text {no full/empty buffers }} .
$$

Accordingly, a state with $i$ buffers that are either full or empty achieves an outage probability equal to $\bar{p}_{2 K-i}$, as $i$ relays provide only one link in the link-selection process. Therefore, the total outage probability is approximated as

$$
\begin{aligned}
P_{\mathrm{out}} & =\sum_{i=1}^{(L+1)^{K}} \pi_{i} \nu_{i} \\
& =\left(\frac{L-1}{L+1}\right)^{K} \bar{p}_{2 K}+\sum_{i=1}^{K} \frac{2^{i}\left(\begin{array}{c}
K \\
i
\end{array}\right)(L-1)^{K-i}}{(L+1)^{K}} \bar{p}_{2 K-i} .
\end{aligned}
$$

For the extreme case with infinite buffer sizes $L \rightarrow \infty$, the outage probability is simplified to

$$
\begin{aligned}
P_{\text {out }}^{\infty} & =\lim _{L \rightarrow \infty}[\underbrace{\left(\frac{L-1}{L+1}\right)^{K}}_{\rightarrow 1} \bar{p}_{2 K}+\sum_{i=1}^{K} \underbrace{\frac{2^{i}\left(\begin{array}{c}
K \\
i
\end{array}\right)(L-1)^{K-i}}{(L+1)^{K}}}_{\rightarrow 0} \bar{p}_{2 K-i}] \\
& =\bar{p}_{2 K} .
\end{aligned}
$$

The above expression shows that for infinite buffer sizes, the max - link relay selection policy always selects between $2 K$ available links (all the links become available) and therefore it ensures a full diversity equal to two times the number of the relays. Simulation results given in the next Section corroborate our analysis, since they demonstrate that for large (but finite) values of $L$ the actual outage probability closely approximates the above bound.

\section{NumericAl Results}

The simulation system follows the system model described in Section II and the relay selection policies considered are: the non-selection policy (where a fixed relay is selected for relaying), the $\max -\min$ relay selection, the $\max -\max$ relay selection (lowest bound) and the investigated max - link relay selection; the selection bound which refers to an ideal max - link selection scheme where all the channel links are always available for selection is used as a reference scheme. In Fig. 4 we plot the outage probability versus the SNR for a simulation setting with $K=2$ relays, $L=2$ and $r_{0}=1 \mathrm{BPCU}$. As it can be seen both the max - min and the max-max selection policies achieve a diversity gain equal to the number of relays (e.g., 2), while the max - max (bound) scheme offers an additional coding gain equal to 1.5 $\mathrm{dB}$ in comparison to the max - min scheme at high SNRs; this observation is in line with the analysis presented in [10]. On the other hand the proposed max - link selection policy also achieves a diversity gain equal to $K=2$ and outperforms the $\max -\max$ (bound) policy with a gain of about $4 \mathrm{~dB}$. It is worth noting that this gain concerns the optimal max - max 


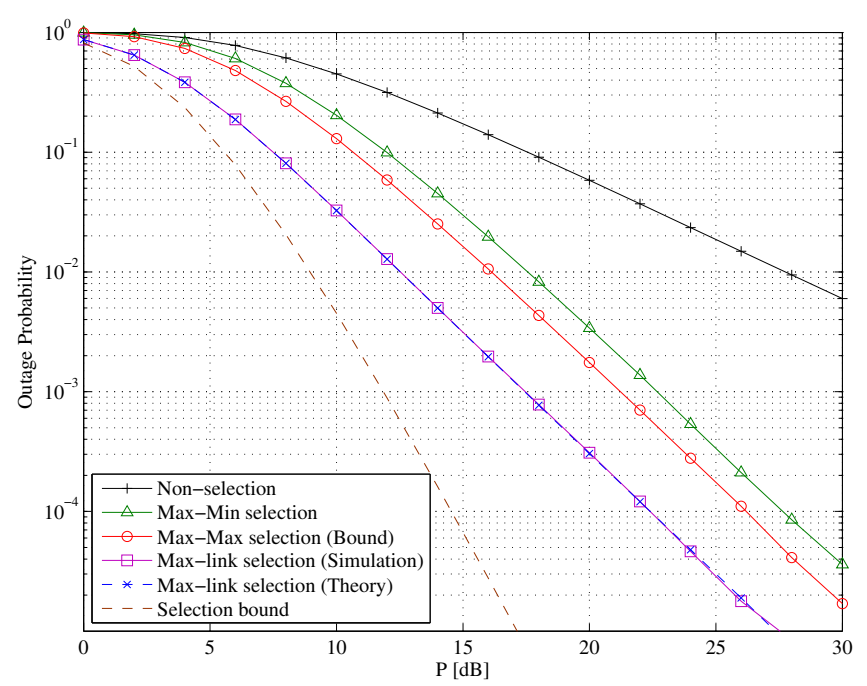

Fig. 4. Outage probability performance versus $\operatorname{SNR}(P)$ for a simulation setting with $K=2$ relays, $L=2$ and $r_{0}=1$ BPCU.

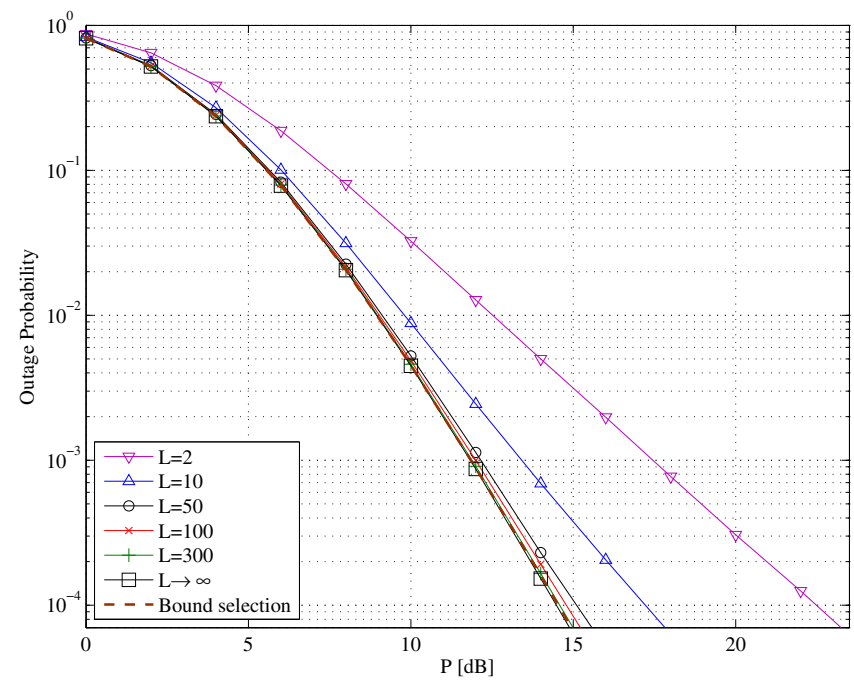

Fig. 5. Outage probability performance versus SNR $(P)$ for max-link relay selection with $K=2$ relays, $L=2,10,50,100,300, \infty$ and $r_{0}=1$ BPCU.

policy (lowest bound) and therefore the gain for a practical version of the max - max should be higher. This result shows that the max - link selection strategy uses more efficiently the existence of the relay buffers and significantly outperforms the existing selection schemes. However, it is worth noting that for the assumed buffer size $L=2$, the max - link does not provide a further diversity gain in comparison to the $\max -\min$ and $\max -\max$ schemes due to the small buffer size; on the other hand the selection bound provides a diversity gain equal to $2 K$. Furthermore, Fig. 4 depicts the theoretical outage probability performance that yields from our theoretical framework that has been presented in Section IV. We can see that the theoretical curve (Eq. (14)) perfectly matches to our simulation results and this observation validates the accuracy of our analysis.

Fig. 5 focuses on the max - link selection scheme and shows the impact of the buffer size on the achieved diversity gain. More specifically, Fig. 5 plots the outage probabil-

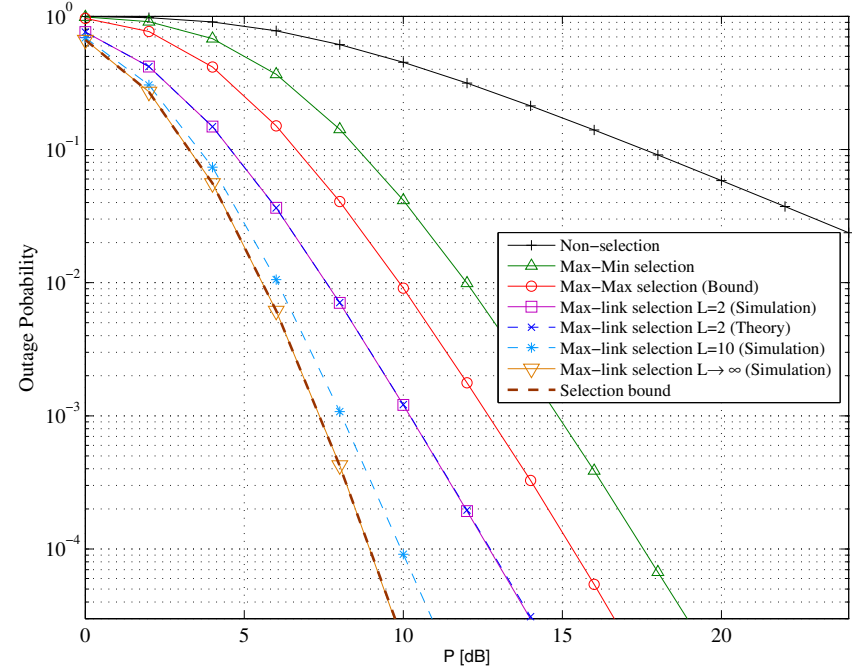

Fig. 6. Outage probability performance versus SNR $(P)$ for $\max$-link relay selection with $K=4$ relays, $L=2,10, \infty$ and $r_{0}=1 \mathrm{BPCU}$.

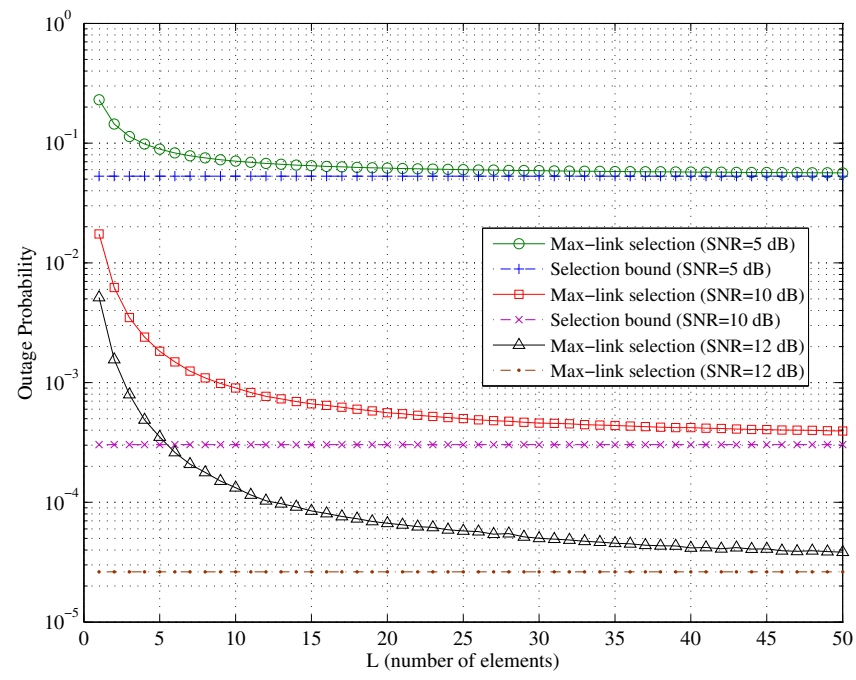

Fig. 7. Outage probability performance versus the size of the relays' buffers $(L)$ for $\max$-link relay selection with $K=3$ relays, $P=5,10,12 \mathrm{~dB}$ (SNR) and $r_{0}=1$ BPCU.

ity performance versus the SNR for different buffer sizes $(L=2,10,50,100, \infty)$ and for the previously used simulation parameters ( $K=2$ relays, $\left.r_{0}=1 \mathrm{BPCU}\right)$. As can be seen, as the buffer size increases the outage probability performance is improved and approaches the selection bound that offers a diversity gain equal to 4 . For a small increase of the buffer size (e.g., $L=10$ packets), the max - link relay selection achieves a diversity gain higher than 2 (the slope of the curve becomes steeper) and therefore it significantly outperforms its previous version with $L=2$ (i.e., $5 \mathrm{~dB}$ gain for an outage probability equal to $10^{-3}$ ). Furthermore, as the size of the buffers increases the diversity order is continuously improved and for $L=100$ it approximates the selection bound and achieves a full diversity equal to 4 ; for the ideal case of $L \rightarrow \infty$, the max - link relay selection policy matches the selection bound, an observation that follows the discussion presented in Section V-B. These remarks reveal the potential of the proposed max - link selection and shows that a quite 


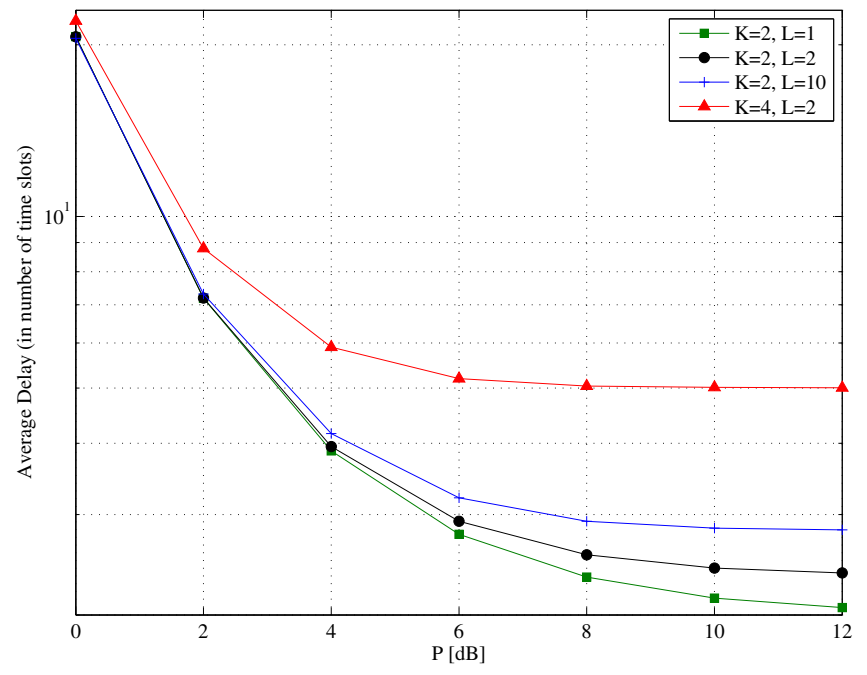

Fig. 8. Average delay in number of time slots versus SNR for different system configurations; $K=2,4$ relays, $L=1,2,10$ and $r_{0}=1$ BPCU.

small buffer size (low complexity) can double the diversity gain in comparison to the existing relay selection schemes. In Fig. 6 we plot the outage probability performance versus the SNR for a simulation setup with $K=4$ relays $L=2,10, \infty$, $r_{0}=1 \mathrm{BPCU}$ and all the considered relay selection schemes. As can be seen the curves follow our previous remarks and validate the observation that the max - link outperforms all the existing relay selection policies and that as the size of the buffers increases, it approaches the selection bound and a diversity order equal to 8 .

In Fig. 7, we plot the outage probability performance of the max - link relay selection scheme versus the size of the relays' buffers $(L)$ for a simulation setup with $K=3$ relays, $r_{0}=1 \mathrm{BPCU}$ and $P=5,10,12 \mathrm{~dB}$; the range of the buffer size $L$ is [1 50] which corresponds to practical implementations with low complexity. As can be seen, as the buffer size is increased, the achieved performance approximates the selection bound. In addition we can see that a relatively small size of buffer is sufficient to achieve the optimal outage performance (e.g., $L=40$ for $P=10 \mathrm{~dB}$ ); however as the SNR increases and the optimal outage probability is decreased, the required value of $L$ is increased (e.g., $L=15$ for $P=5$ $\mathrm{dB}$ and $L=40$ for $P=10 \mathrm{~dB}$ ). Finally, Fig. 8 plots the average time delay in number of time slots versus the SNR for different system configurations in order to give an idea about this important metric. It can be seen that as the number of relays increases, the probability to select a specific relay for transmission decreases and thus the average delay increases. In addition, as the buffer size increases, the probability to have a full relay and thus to select a specific relay decreases, which results in an increase of the average delay. By tolerating a higher delay, the diversity performance improves as the number of relays $K$ or buffer size $L$ increases, as shown in Figs. 5 and 6.

\section{CONCLUSIONS}

In this paper, we investigated a max - link relay selection policy for cooperative networks with finite buffers at the relay nodes. Unlike conventional approaches, the proposed scheme fully exploits the buffering capability at the relays and schedules transmissions only through the strongest available channel link in order to extract further diversity benefits. A methodology that models the buffer evolution as a $\mathrm{MC}$ has been adopted and the achieved outage probability performance of the system has been derived in closed form. We conclude that the max - link relay selection scheme outperforms previously reported schemes by providing a significant coding gain for small buffer sizes, while it ensures a diversity gain approaching the upper-bound of two-times the number of relays for large buffer sizes. The max - link relay selection scheme is introduced as an efficient cooperative technique for systems with multiple relays and no latency constraints. Numerous possible extensions of this work are possible and clearly suggested, namely, inclusion of an asymmetric channel model, partial channel knowledge and consideration of delay constraints.

\section{APPENDIX A}

\section{PROOF OF LEMMA 1}

Since the MC considered is irreducible and aperiodic, then there exists a unique solution $\boldsymbol{\pi}=\left(\begin{array}{llll}\boldsymbol{\pi}_{1} & \boldsymbol{\pi}_{2} & \ldots & \boldsymbol{\pi}_{(L+1)^{K}}\end{array}\right)^{T}$ such that

$$
\begin{aligned}
\mathbf{A} \boldsymbol{\pi} & =\boldsymbol{\pi}, \\
\sum_{i=1}^{(L+1)^{K}} \boldsymbol{\pi}_{i} & =1 .
\end{aligned}
$$

The solution can be easily found by solving the set of linear equations. In this case, (26) is written as

$$
\mathrm{B} \pi=\mathbf{b},
$$

where $\boldsymbol{b}=\left(\begin{array}{llll}1 & 1 & \ldots & 1\end{array}\right)^{T}$ and $\mathbf{B}_{i, j}=1, \forall i, j$. Then, by adding (25) and (27), we obtain that

$$
\mathbf{A} \boldsymbol{\pi}-\boldsymbol{\pi}+\mathbf{B} \boldsymbol{\pi}=\mathbf{b} \Rightarrow(\mathbf{A}-\mathbf{I}+\mathbf{B}) \boldsymbol{\pi}=\mathbf{b} .
$$

Now, we have to show that the inverse of $(\mathbf{A}-\mathbf{I}+\mathbf{B})$ exists, and hence $\boldsymbol{\pi}=(\mathbf{A}-\mathbf{I}+\mathbf{B})^{-1} \mathbf{b}$. Let $\mathbf{y}$ be a row vector such that

$$
\mathbf{y}(\mathbf{A}-\mathbf{I}+\mathbf{B})=\mathbf{0} .
$$

It is sufficient to show that $\mathbf{y}=\mathbf{0}$. Multiplying (29) by $\boldsymbol{\pi}$, we have

$$
\mathbf{y}(\mathbf{A}-\mathbf{I}+\mathbf{B}) \boldsymbol{\pi}=\mathbf{y}(\mathbf{A} \boldsymbol{\pi}-\boldsymbol{\pi}+\mathbf{B} \boldsymbol{\pi})=\mathbf{y b}=\mathbf{0} .
$$

Since $\mathbf{b}$ has strictly positive entries and $\mathbf{y b}=\mathbf{0}$, we conclude that $\mathbf{y}=\mathbf{0}$. We have shown that there exists a unique solution and that it is given by (12)

$$
\boldsymbol{\pi}=(\mathbf{A}-\mathbf{I}+\mathbf{B})^{-1} \mathbf{b} .
$$

For completeness, we show that $\boldsymbol{\pi}$ is positive. Since $\mathbf{A}$ is nonnegative and irreducible matrix, and $\mathbf{A}<\mathbf{B}$ then $\mathbf{A}+\mathbf{B}$ is positive and irreducible and hence, $\mathbf{A}-\mathbf{I}+\mathbf{B}$ is positive and irreducible. As a result, from Perron-Frobenius theorem [23], $(\mathbf{A}-\mathbf{I}+\mathbf{B})^{-1}$ exists and it is positive componentwise. Therefore, since $\mathbf{b}$ is positive component-wise, then $\pi$ is positive. 


\section{APPENDIX B \\ PROOF OF LEMMA 2}

Since the chain is reversible, $\boldsymbol{\pi}_{i} \mathbf{A}_{i, j}=\boldsymbol{\pi}_{j} \mathbf{A}_{j, i}$. Solving for $\boldsymbol{\pi}_{j}$ for states $j$ that communicate with state $i$, we have

$$
\boldsymbol{\pi}_{j}=\boldsymbol{\pi}_{i} \frac{\mathbf{A}_{i, j}}{\mathbf{A}_{j, i}}
$$

For a state $k$ with which state $i$ communicates via state $j$ we have

$$
\boldsymbol{\pi}_{k}=\boldsymbol{\pi}_{j} \frac{\mathbf{A}_{j, k}}{\mathbf{A}_{k, j}}=\boldsymbol{\pi}_{i} \frac{\mathbf{A}_{i, j} \mathbf{A}_{j, k}}{\mathbf{A}_{k, j} \mathbf{A}_{j, i}} .
$$

In the general form, this can be written as

$$
\boldsymbol{\pi}_{j}=\boldsymbol{\pi}_{i} \frac{\prod_{k \in \Xi\left(i, \Theta_{j}\right)} \mathbf{A}_{i, k}}{\prod_{m \in \Xi\left(j, \Theta_{i}\right)} \mathbf{A}_{j, m}} .
$$

Substituting (30) in (26)

$$
\begin{aligned}
& \boldsymbol{\pi}_{i} \sum_{j=1}^{(L+1)^{K}} \frac{\prod_{k \in \Xi\left(i, \Theta_{j}\right)} \mathbf{A}_{i, k}}{\prod_{m \in \Xi\left(j, \Theta_{i}\right)} \mathbf{A}_{j, m}}=1 \\
& \Rightarrow \boldsymbol{\pi}_{i}=\left(\sum_{j=1}^{(L+1)^{K}} \frac{\prod_{k \in \Xi\left(i, \Theta_{j}\right)} \mathbf{A}_{i, k}}{\prod_{m \in \Xi\left(j, \Theta_{i}\right)} \mathbf{A}_{j, m}}\right)^{-1} .
\end{aligned}
$$

Hence, we are able to easily find $\boldsymbol{\pi}_{i}$ and consequently $\boldsymbol{\pi}_{j}$ for all states.

\section{REFERENCES}

[1] J. N. Laneman, D. N. C. Tse, and G. W. Wornell, "Cooperative diversity in wireless networks: efficient protocols and outage behavior," IEEE Trans. Inf. Theory, vol. 50, pp. 3062-3080, Dec. 2004.

[2] A. Bletsas, A. Khisti, D. Reed, and A. Lippman, "A simple cooperative diversity method based on network path selection," IEEE J. Sel. Areas Commun., vol. 24, pp. 659-672, Mar. 2006.

[3] H. A. Suraweera, M. Soysa, C. Tellambura, and H. K. Garg, "Performance analysis of partial relay selection with feedback delay," IEEE Signal Process. Lett., vol. 17, pp. 531-534, June 2010.

[4] A. S. Ibrahim, A. K. Sadek, W. Su, and K. J. Ray Liu, "Cooperative communications with relay-selection: when to cooperate and whom to cooperate with?" IEEE Trans. Wireless Commun., vol. 7, pp. 2814-2827, July 2008.

[5] I. Krikidis, J. S. Thompson, S. McLaughlin, and N. Goertz, "Max-Min relay selection for legacy amplify-and-forward systems with interference," IEEE Trans. Wireless Commun., vol. 8, pp. 3016-3027, June 2009.

[6] W. -J. Huang, Y. -W. Hong, and C. -C. J. Kuo, "Lifetime maximization for amplify-and-forward cooperative networks," IEEE Trans. Wireless Commun., vol. 7, pp. 1800-1805, May 2008.

[7] Z. Ding, K. Leung, D. L. Goeckel, and D. Towsley, "Opportunistic relaying for secrecy communications: cooperative jamming vs relay chatting," IEEE Trans. Wireless Commun., vol 10, pp. 1725-1729, June 2011.

[8] A. Bletsas, H. Shin, and M. Z. Win, "Cooperative communications with outage-optimal opportunistic relaying," IEEE Trans. Wireless Commun., vol. 6, pp. 3450-3460, Sep. 2007.

[9] D. S. Michalopoulos and G. K. Karagiannidis, "Performance analysis of single relay selection in Rayleigh fading," IEEE Trans. Wireless Commun., vol. 7, pp. 3718-3724, Oct. 2008.

[10] A. Ikhlef, D. S. Michalopoulos, and R. Schober, "Buffers improve the performance of relay selection," in Proc. 2011 IEEE Global Commun. Conf., pp. 1-6.

[11] N. Zlatanov, R. Schober, and P. Popovski, "Throughput and diversity gain of buffer-aided relaying," in Proc. 2011 IEEE Global Commun. Conf., pp. 1-6.

[12] R. Wang, V. K. N. Lau, and H. Huang, "Opportunistic buffered decode-wait-and-forward (OBDWF) protocol for mobile wireless relay networks," IEEE Trans. Wireless Commun., vol. 10, pp. 1224-1231, Apr. 2011.
[13] A. K. Sadek, K. J. Ray Liu, and A. Ephremides, "Cognitive multiple access via cooperation: protocol design and performance analysis," IEEE Trans. Inf. Theory, vol. 53, pp. 3677-3696, Oct. 2007.

[14] N. B. Mehta, V. Sharma, and G. Bansal, "Performance analysis of a cooperative system with rateless codes and buffered relays," IEEE Trans. Wireless Commun., vol. 10, pp. 1069-1081, Apr. 2011.

[15] B. Xia, Y. Fan, J. S. Thompson, and H. V. Poor, "Buffering in a threenode relay network," IEEE Trans. Wireless Commun., vol. 7, pp. 44924496, Nov. 2008.

[16] L. Ding, M. Tao, F. Yang, and W, Zhang, "Joint scheduling and relay selection in one- and two-way relay networks with buffering," in Proc. 2009 IEEE Int. Conf. Commun., pp. 1-5.

[17] K.-S. Hwang, Y.-C. Ko, and M.-S. Alouini, 'Performance analysis of two-way amplify-and-forward relaying with adaptive modulation over multiple relay network," IEEE Trans. Wireless Commun., vol. 59, pp. 402-406, Feb. 2011.

[18] S. Yang and J.-C. Belfiore, "Towards the optimal amplify-and-forward cooperative diversity scheme," IEEE Trans. Inf. Theory, vol. 53, pp. 3114-3126, Sep. 2007.

[19] I. Krikidis, J. S. Thompson, S. McLaughlin, and N. Goertz, "Amplifyand-forward with partial relay selection," IEEE Commun. Lett., vol. 12, pp. 235-237, Apr. 2008.

[20] D. Benevides da Costa and S. Aissa, "End-to-end performance of dualhop semi-blind relaying systems with partial relay selection," IEEE Trans. Wireless Commun., vol. 8, pp. 4306-4315, Aug. 2009.

[21] D. Benevides da Costa and S. Aissa, "Performance analysis of relay selection techniques with clustered fixed-gain relays," IEEE Signal Process. Lett., vol. 17, pp. 201-204, Feb. 2010.

[22] J. R. Norris, Markov Chains. Cambridge University Press, 1998.

[23] A. Berman and R. J. Plemmons, Nonnegative Matrices in the Mathematical Sciences. Society of Industrial and Applied Mathematics, 1994.

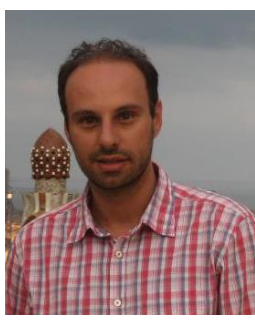

Ioannis Krikidis ( $\mathrm{S}^{\prime} 03-\mathrm{M}^{\prime} 07$ ) was born in Athens, Greece, in 1977. He received the diploma in Computer Engineering from the Computer Engineering and Informatics Department (CEID) of the University of Patras, Greece, in 2000, and the M.Sc and $\mathrm{Ph} . \mathrm{D}$. degrees from Ecole Nationale Supérieure des Télécommunications (ENST), Paris, France, in 2001 and 2005, respectively, all in electrical engineering.

From 2006 to 2007 he worked, as a Post-Doctoral researcher, with ENST, Paris, France, and from 2007 to 2010 he was a Research Fellow in the School of Engineering and Electronics at the University of Edinburgh, Edinburgh, UK. During summer of 2008 and spring of 2009, he was visiting researcher at the University of Notre Dame, IN, USA and the University of Maryland, USA, respectively. He has been recently elected as an Assistant Professor at CEID, University of Patras, Greece, while he is currently a Visiting Assistant Professor at the Department of Electrical and Computer Engineering, University of Cyprus, Nicosia, Cyprus. His current research interests include information theory, wireless communications, cognitive radio and secrecy communications. Dr. I. Krikidis is a member of the Technical Chamber of Greece.

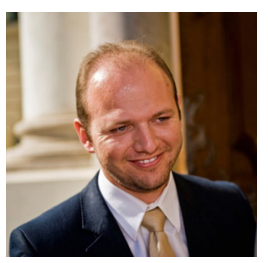

Themistoklis Charalambous received his BA and M.Eng in Electrical and Information Sciences from Cambridge University in 2005. He pursued his Ph.D. in the Control Laboratory, of the Engineering Department, Cambridge University in 2009. He worked as a Research Associate at Imperial College London and as a Visiting Lecturer at the Department of Electrical and Computer Engineering, University of Cyprus. He is currently working at the Automatic Control Lab of the School of Electrical Engineering at the Royal Institute of Technology (KTH) as a Research Associate. His research involves cooperative control, distributed decision making, game theory, and control to various resource allocation problems in complex and networked systems, such as wireless ad hoc networks. 


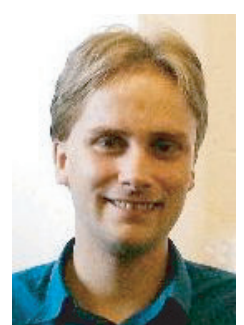

John S. Thompson received his BEng and Ph.D. degrees from the University of Edinburgh in 1992 and 1996, respectively. From July 1995 to August 1999, he worked as a postdoctoral researcher at Edinburgh, funded by the UK Engineering and Physical Sciences Research Council (EPSRC) and Nortel Networks. He was appointed as a lecturer at what is now the School of Engineering at the University of Edinburgh in 1999. He was recently promoted to a personal chair in Signal Processing and Communications.

Dr John S. Thompson's research interests currently include energy efficient communications systems, antenna array techniques and multi-hop wireless communications. He has published over 200 papers to date including a number of invited papers, book chapters and tutorial talks, as well as coauthoring an undergraduate textbook on digital signal processing. He is overall project leader for the $£ 1.2 \mathrm{M}$ EPSRC Islay project involving four universities which investigates efficient hardware implementation of complex algorithms. $\mathrm{He}$ is also the academic deputy leader for the £2M EPSRC/Mobile VCE Green Radio project, which involves a number of international communication companies. He is currently editor-in-chief of the IET Signal Processing journal and was a technical programme co-chair for the Globecom conference in Miami in December 2010. 